CERN-TH/97-150

hep-ph/9707248

\title{
The Contribution of $B_{c}$ Mesons to
}

\section{THE SEARCH FOR $B^{+} \rightarrow \tau^{+} \nu_{\tau}$ DECAYS AT LEP}

\author{
Michelangelo L. MANGANO円 \\ CERN, TH Division, Geneva, Switzerland \\ mlm@vxcern.cern.ch \\ S.R. SLABOSPITSKY \\ Institute for High Energy Physics, Protvino, Moscow Region, 142284 Russia \\ slabospitsky@mx. ihep.su
}

\begin{abstract}
We study the contribution of $B_{c}$ mesons to the search for $B \rightarrow \tau \nu_{\tau}$ decays. We find that at LEP the contributions from $B_{u}$ and $B_{c}$ mesons can be comparable. This observation can have a relevant impact on the extraction of constraints on new physics (such as chargedHiggs contributions) from current LEP limits on $B \rightarrow \tau \nu$ final states. Inclusion of the $B_{c}$ contribution can reduce the current L3 limit on $\tan \beta / M_{H}$ from $0.38 \mathrm{GeV}^{-1}$ (90\%CL) down to $0.27 \mathrm{GeV}^{-1}(90 \% \mathrm{CL})$.
\end{abstract}

CERN-TH/97-150

August 21, 2018

\footnotetext{
${ }^{1}$ On leave of absence from INFN, Pisa, Italy
} 
The study of purely leptonic decays of heavy charged mesons $P^{+}\left(\right.$e.g. $\left.D^{ \pm}, D_{s}^{ \pm}, B^{ \pm}, \ldots\right)$

$$
P^{+}(Q \bar{q}) \rightarrow \ell^{+} \nu_{\ell}
$$

is of particular interest, due to their sensitivity to both the meson decay constants $f_{P}$ and the

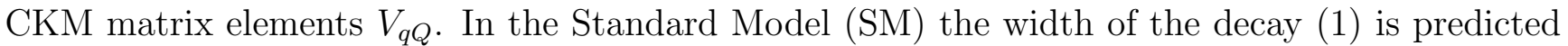
to be (here we assume a zero value for neutrino mass):

$$
\Gamma_{S M}\left(P^{+} \rightarrow \ell^{+} \nu_{\ell}\right)=\frac{G_{F}^{2}}{8 \pi}\left|V_{Q q}\right|^{2} f_{P}^{2} M_{P} m_{\ell}^{2}\left(1-\frac{m_{\ell}^{2}}{M_{P}^{2}}\right)^{2},
$$

where $G_{F}$ is the Fermi coupling constant and $M_{P}$ and $m_{\ell}$ are the masses of the pseudoscalar meson $P$ and of the charged lepton $\ell^{+}$, respectively.

The widths of such decays are also very sensitive to the possible presence of physics beyond the SM. For example, in models with two Higgs doublets the decay width given in eq. (2) acquires the additional factor due the annihilation via a charged Higgs $\left(H^{ \pm}\right)$[1]:

$$
\Gamma_{H^{ \pm}}\left(P^{+} \rightarrow \ell^{+} \nu_{\ell}\right)=\Gamma_{S M}\left(P^{+} \rightarrow \ell^{+} \nu_{\ell}\right) \times\left(1-\frac{\tan ^{2} \beta}{M_{H}^{2}} M_{P}^{2}\right)^{2},
$$

where $\tan \beta$ is the ratio of the vacuum expectation values for the Higgs fields and $M_{H}$ is the mass of the charged Higgs boson. Similarly, a possible admixture of a right-handed $(V+A)$ current to the standard $(V-A)$ current could also lead to modifications of eq. (2) (see [2, 3]).

Because of the strong helicity suppression, $e$ and $\mu$ leptonic decays of $B$ mesons are very far from being observed. On the contrary, the statistics available today at CLEO and LEP or becoming available in the very near future at the $B$-factories should allow the first observation of the $B \rightarrow \tau \nu_{\tau}$ decay mode. Notice that even in this case, however, the $B_{u} \rightarrow \tau \nu_{\tau}$ decay has a very small branching ratio, of the order of few $\times 10^{-5}$. The current results of these searches at low energies (in the reaction $\Upsilon(4 S) \rightarrow B^{+} B^{-}$[四, 可]) as well as at the energy of the LEP collider [7, [6] are collected in Table 1. The recent measurement by L3 [7], based on a fraction of the full data sample accumulated during the LEP running, set an upper limit on the $B \rightarrow \tau \nu_{\tau}$ branching ratio (BR) which is within less than an order of magnitude from the SM estimate. This limit is nevertheless already sufficient to set interesting constraints on the allowed values of $\tan \beta / M_{H}$ 团 $\mathrm{Z}$

In this note we point out that for measurements carried out at energies well above the threshold for the production of $b \bar{b}$ pairs, production and decay of $B_{c}$ mesons can give a substantial contribution to $\tau \nu_{\tau}$ final states 5 This observation strengthens the current limits from LEP on $B R\left(B_{u} \rightarrow \tau \nu\right)$ and, as a result, on the possible presence of new physics. As we shall see, the rate for $Z^{0} \rightarrow B_{c} X \rightarrow \tau \nu X$ could well be larger than that for $Z^{0} \rightarrow B_{u} X \rightarrow \tau \nu X$, making this process a possible discovery channel for the as yet unobserved $B_{c}$ meson.

Bound states of a $b$ and $\bar{c}$ quark pair (the $B_{c}$ meson) have never been observed. They represent interesting objects because their production and decay properties are expected to be calculable

\footnotetext{
${ }^{2}$ In a recent paper $[8]$, the prospects for the extraction of tight constraints on this parameters using $B \rightarrow D \tau \nu$ decays have also been considered.

${ }^{3}$ This observation has certainly been made already in the past. We recently learned, for example, that M. Neubert made this remark in several occasions. To our knowledge, however, a detailed study of its consequences, in the light of the current measurements from LEP, has never been documented.
} 


\begin{tabular}{|c||c|c|c|}
\hline Exp. & energy & $\operatorname{Br}(B \rightarrow \tau \nu)$ & $\frac{\tan \beta}{M_{H}}, \mathrm{GeV}^{-1}$ \\
\hline ARGUS [4] & $\Upsilon(4 S)$ & $<1.04 \times 10^{-2}$ & $<0.69$ \\
\hline CLEO [5] & $\Upsilon(4 S)$ & $<2.2 \times 10^{-3}$ & $<0.489$ \\
\hline ALEPH [6] & $Z$-boson & $<1.8 \times 10^{-3}$ & $<0.468$ \\
\hline L3 [7] & $Z$-boson & $<5.7 \times 10^{-4}$ & $<0.38$ \\
\hline
\end{tabular}

Table 1: Experimental upper limits for the branching ratio of $B^{ \pm} \rightarrow \tau^{ \pm} \nu_{\tau}$ decay and the evaluated constraint for charged Higgs boson parameters.

within QCD. Typical non-perturbative parameters such as the decay constant $f_{B_{c}}$ can be calculated by using, for example, potential models or QCD sum rules (see for example the review in ref. [9] and the references therein).

The relative fraction of $\tau \nu$ final states coming from $B_{c}$ and $B_{u}$ production is given by:

$$
\frac{N_{B_{c}}}{N_{B_{u}}}=\frac{f\left(b \rightarrow B_{c}\right)}{f\left(b \rightarrow B_{u}\right)}\left|\frac{V_{c b}}{V_{u b}}\right|^{2}\left(\frac{f_{B_{c}}}{f_{B_{u}}}\right)^{2} \frac{m_{B_{c}}}{m_{B_{u}}} \frac{\tau_{B_{c}}}{\tau_{B_{u}}} \frac{\left(1-\frac{m_{\tau}^{2}}{m_{B_{c}}^{2}}\right)^{2}}{\left(1-\frac{m_{\tau}^{2}}{m_{B_{u}}^{2}}\right)^{2}}
$$

where $\tau_{B_{c}}$ and $\tau_{B_{u}}$ refer to the $B_{c}$ and $B_{u}$ lifetimes, and the factor $f\left(b \rightarrow B_{c}\right)\left(f\left(b \rightarrow B_{u}\right)\right)$ is the inclusive probability that a $b$ quark hadronizes into a $B_{c}\left(B_{u}\right)$ meson. These factors include transitions in which the $b$ quark hadronizes into excited $B_{u}$ and $B_{c}$ states, which then decay strongly into the stable scalar mesons. While $f\left(b \rightarrow B_{c}\right)$ is estimated to be a small number, of the order of $0.1 \%$, the large ratio $\left(V_{c b} / V_{u b}\right)$ can largely compensate for the suppressed $B_{c}$ production rate.

Before turning to the numerical estimate of the $B_{c}$ contribution to the $\tau \nu$ rate, we discuss the values and potential correlations of the parameters present in eq. (4). We start from the parameters relative to the $B_{u}$ system. Mass and lifetime are known today with good accuracy [10]:

$$
m_{B_{u}}=5.2789 \pm 0.0018 \mathrm{GeV}, \quad \tau_{B_{u}}=(1.62 \pm 0.06) \times 10^{-12} \mathrm{~s} .
$$

The probability $f\left(b \rightarrow B_{u}\right)$ is known at LEP with accuracy better than $10 \%$ [10]:

$$
f\left(b \rightarrow B_{u}\right)=0.378 \pm 0.022 .
$$

The value of the decay constant $f_{B_{u}}$ has never been measured, but estimates based on lattice calculations have achieved a good degree of accuracy, and give [11]:

$$
f_{B_{u}}=175 \pm 25 \mathrm{MeV}
$$

Of course the value of $f_{B_{u}}$ is exactly one of the parameters which one would like to extract from the measurement of the leptonic $B_{u}$ decay. As we will show, doing this with sufficient accuracy requires the use of a sample of $B_{u}$ mesons without a $B_{c}$ contamination. Use of the central value 
for the above parameters, in addition to using the central value of $V_{u b}=0.0033 \pm 0.0008$, gives the following

$$
B R\left(B_{u} \rightarrow \tau \nu_{\tau}\right)=5.8 \times 10^{-5}
$$

The only parameter relative to the $B_{c}$ meson which is expected to be known with good accuracy from the current calculations is the mass [9, 13]:

$$
m_{B_{c}}=6.275 \pm 0.040 \mathrm{GeV} .
$$

A recent thorough analysis of the $B_{c}$ lifetime, performed within NRQCD and including nonrelativistic corrections up to order $v^{2}$, has been carried out in ref. [12]. The result is given by:

$$
\tau_{B_{c}}=(0.55 \pm 0.15) \times 10^{-12} \mathrm{~s},
$$

where the quoted uncertainty is dominated by the limited knowledge of the charm and bottom quark masses, and to a lesser degree by the uncertainty in the strange quark mass and in $V_{c b}$. Because of the almost complete cancellation between the weak-annihilation rate and the effect of Pauli-interference diagrams, no significant uncertainty on the lifetime arises from the limited knowledge of the decay constant $f_{B_{c}}$ [12]. This has been calculated both in potential models [9, 13] and by use of sum-rules [14. A complete review of these results and a comprehensive bibliography can be found in ref. [9]. From this review we extract the following range of values for $f_{B_{c}}$ :

$$
f_{B_{c}}=450 \pm 100 \mathrm{MeV} .
$$

The transition probability $f\left(b \rightarrow B_{c}\right)$ at LEP has been evaluated by several authors [15]. It is in principle calculable from QCD once the value of the meson wave function is known. The leading production mechanism is through the emission of an off-shell gluon from the $b$ quark, with the gluon splitting into a $c \bar{c}$ pair and the $b \bar{c}$ system binding into the $B_{c}$ or excited states thereof. Several uncertainties are present in this calculation. For example, NLO QCD corrections are not known, and the result is very sensitive on the choice of renormalization scale. Likewise, the wave functions of the ground state state and of the excited states are not known with high accuracy, as discussed above. We shall therefore leave $f\left(b \rightarrow B_{c}\right)$ a free parameter, varying it in the range suggested by the envelope of the available calculations $\left(0.02 \%<f\left(b \rightarrow B_{c}\right)<0.1 \%\right)$ and studying the dependence of our results on it.

The final parameter which is needed to determine the quantity in eq. (四) is the ratio $V_{u b} / V_{c b}$. The value for this ratio found in the RPP [10] is:

$$
\frac{V_{u b}}{V_{c b}}=0.08 \pm 0.02
$$

Using the central values of the parameters discussed so far, we obtain the following result:

$$
\frac{N_{B_{c}}}{N_{B_{u}}}=1.2\left[\frac{f\left(b \rightarrow B_{c}\right)}{10^{-3}}\right] .
$$

The upper and lower values which can be obtained by taking the extreme ranges quoted for the parameters $f_{B_{c}}$ and $\tau_{B_{c}}$ are:

$$
\left(\frac{N_{B_{c}}}{N_{B_{u}}}\right)_{\min }=0.52\left[\frac{f\left(b \rightarrow B_{c}\right)}{10^{-3}}\right]
$$




\begin{tabular}{|c||c|c|c|}
\hline \multicolumn{1}{|c||}{} & \multicolumn{3}{c|}{$\left(\tan \beta / M_{H}\right)_{\min }\left(\mathrm{GeV}^{-1}\right)$} \\
\hline$f\left(b \rightarrow B_{c}\right)$ & low & central & high \\
\hline $2 \times 10^{-4}$ & 0.37 & 0.35 & 0.33 \\
$1 \times 10^{-3}$ & 0.33 & 0.30 & 0.27 \\
\hline
\end{tabular}

Table 2: Lower limits on the ratio $\tan \beta / M_{H}\left(\mathrm{GeV}^{-1}\right)$ obtained by taking into account the contribution of $B_{c}$ mesons to the L3 search for $b \rightarrow \tau \nu$ final states.

and

$$
\left(\frac{N_{B_{c}}}{N_{B_{u}}}\right)_{\max }=2.3\left[\frac{f\left(b \rightarrow B_{c}\right)}{10^{-3}}\right] .
$$

As is seen from eq. (14), even the minimum relative contribution due to $B_{c}$ meson can have a noticeable magnitude $(\sim 50 \%)$. Therefore, this effect should be taken into account when one considers the results of the search and analysis of the $B_{u} \rightarrow \tau \nu$ decay.

To demonstrate the importance of this $B_{c}$ contribution let us analyze the results of the L3 Collaboration, which reported the following upper limit for the branching ration of the $B_{u} \rightarrow \tau^{ \pm} \nu_{\tau}$ decay [7]:

$$
\operatorname{Br}\left(B_{u} \rightarrow \tau \nu\right)<5.7 \times 10^{-4} \quad(90 \% C L) .
$$

This is almost a factor of 10 larger than the SM prediction in eq. (10). In order to assess the impact of the $B_{c}$ production and decay on this measurement, we would need an estimate of the relative tagging efficiency for the secondary vertex of $\tau$-tracks from $B_{c}$ and from $B_{c}$ decays. Since the expected lifetime of the $B_{c}$ is a factor $2 \div 3$ smaller than the lifetime of the $B_{u}$, we expect a possible loss in efficiency, and a dilution of the $B_{c}$ contamination of the $\tau \nu$ signal. We notice that in previous searches for $B_{c}$ production at LEP [19, 20, 21], using $B_{c} \rightarrow \psi \ell \nu$ or $B_{c} \rightarrow \psi \pi$ decays, the changes in efficiency due varying $\tau_{B_{c}}$ in the range $0.4 \div 1.4$ ps have been estimated to be small, of the order of $20 \%$. In absence of a specific study for the $\tau \nu$ final state, we shall assume for definiteness that the $B_{c}$ and $B_{u}$ decays have the same efficiency.

Using the eq. (B) and the result (16) the authors of [7] obtain the following constraint for the contribution due to charged Higgs boson:

$$
\frac{\tan \beta}{M_{H}}\left(B_{u}\right)<0.38 \quad \text { [7]. }
$$

Taking into account the $B_{c}$ meson contribution we can improve this constraint. Our results are shown in Table 2, for several values of $f\left(b \rightarrow B_{c}\right)$ and for extreme choices of the $B_{c}$ system parameters.

In the Fig 1. we exhibit the present limits for $M_{H}$ and $\tan \beta$ parameters of the charged Higgs boson $H^{ \pm}$. In this figure we combine the results from the direct searches of $H^{ \pm}$(CDF Collaboration [16] and DELPHI Collaboration [18]), from the measurements of the $b \rightarrow s \gamma$ transition 


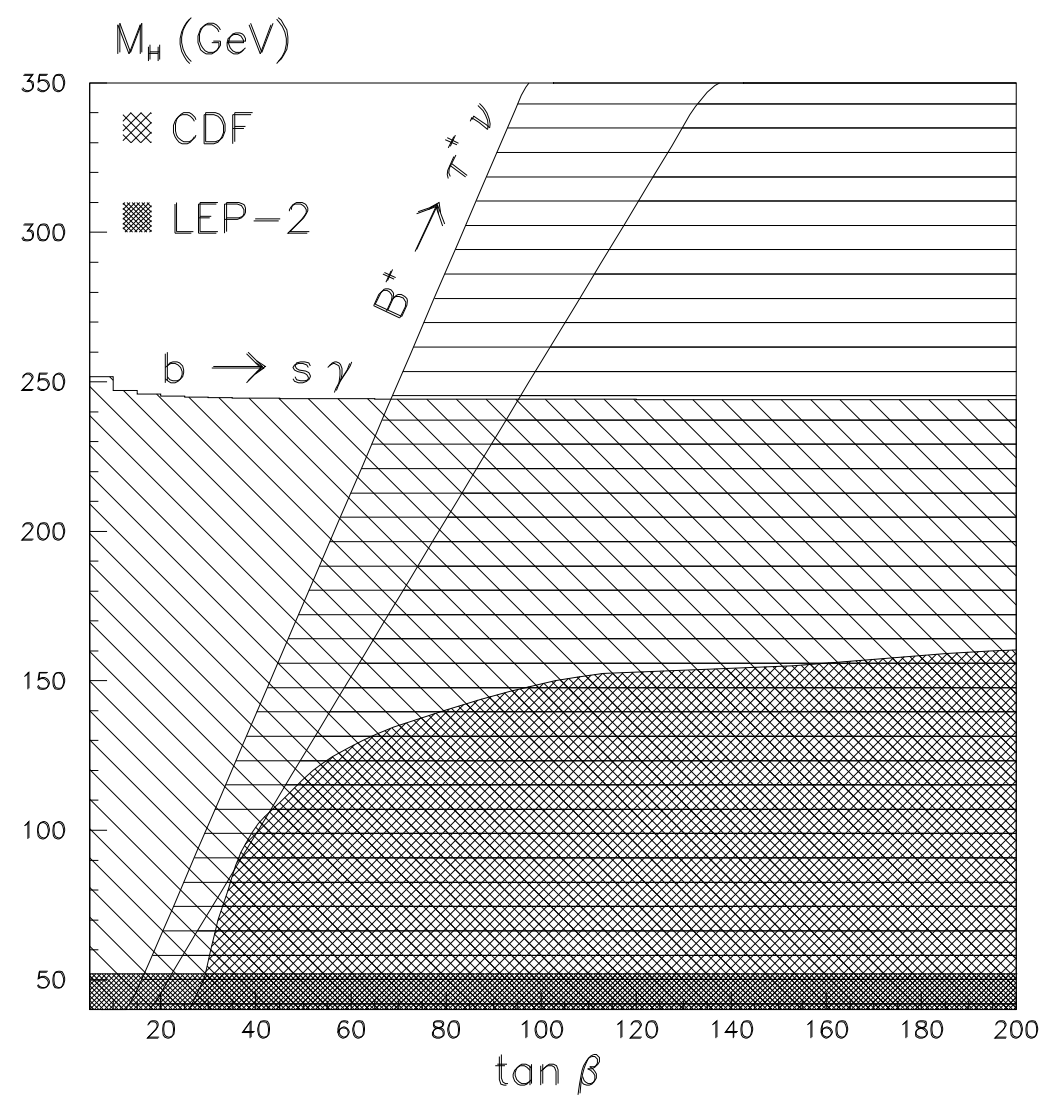

Figure 1: Present limits for $M_{H}$ and $\tan \beta$. The two diagonal lines next to the caption $B^{+} \rightarrow \tau \nu$ refer to the constraints derived from the L3 limit. The right-most line is the L3 limit, the left-most line corresponds to the 0.27 entry in table 2 of this work.

(CLEO Collaboration [17]), and the results from the search for $B \rightarrow \tau \nu$ decay (the right line represents original L3 Collaboration [7] result(17)) while the left line corresponds to this article analysis).

In summary, we discussed in this paper the possible effects of $B_{c}$ production and leptonic decay on current $b \rightarrow \tau \nu$ searches at LEP. We showed that the $B_{c} \rightarrow \tau \nu$ decay can produce a number of $b \rightarrow \tau \nu$ events as large as the $B_{u} \rightarrow \tau \nu$ process. The uncertainties in the production and decay properties of the $B_{c}$ meson are such that no precision measurement of either $f_{B_{u}}$ or $V_{u b}$ can therefore be expected from LEP, or similar high-statistics measurements done at machines producing $b$ quarks well above threshold. These problems will clearly disappear at the forthcoming $B$-factories, where no contribution from $B_{c}$ mesons is possible. We showed that the limits on possible new physics obtained from the LEP measurements, e.g. leptonic decays mediated by charged Higgses, can however be significantly improved when the effect of $B_{c}$ production and decay is accounted for. These improved limits will become more compelling once the leading uncertainties in the determination of the properties of the $B_{c}$ system will be improved. For example the detection of $B_{c}$ candidates in other channels, such as $B_{c} \rightarrow \psi \ell \nu$, should allow to fix 
the main unknown, namely the value of $f\left(b \rightarrow B_{c}\right)$.

Acknowledgements We are grateful to A. Falk, M. Neubert, V.F. Obraztsov and A.A. Sokolov for useful discussions.

\section{References}

[1] W.S. Hou, Phys. Rev. D48 (1993) 2342.

[2] M.B. Voloshin, TPI-MINN-97/08-T, hep-ph/9704278.

[3] D. Du, H. Jin, and Y. Yang, BIHEP-Th/97-007, hep-ph/9705261.

[4] H. Albrecht et al. ARGUS Collaboration, Phys. Lett. B353 (1996) 554.

[5] M. Artuso et al. CLEO Collaboration, Phys. Rev. Lett. 75 (1995) 785.

[6] D. Buskulic et al. ALEPH Collaboration, Phys. Lett. B343 (1995) 444.

[7] M. Acciarri et al. L3 Collaboration, Phys. Lett. B396 (1997) 327.

[8] K. Kiers and A. Soni, hep-ph/9706337.

[9] S.S. Gershtein, V.V. Kiselev, A.K. Likhoded, and A.V. Tkabladze. Phys. Usp. 38 (1995) 1, Usp. Fiz. Nauk 165 (1995) 3 (in russian), hep-ph/9504319; Phys. Rev. D51 (1995) 3613.

[10] R.M. Barnett et al. (Review of Particle Properties), Phys. Rev. D54, Part II (1996) 1.

[11] G. Martinelli, in Proceedings of the 28th International Conference on High Energy Physics, Z. Ajduk and A.K. Wroblewski eds., World Scientific, 1997, Vol.I, p.164.

[12] M. Beneke and G. Buchalla, Phys. Rev. D53 (1996) 4991.

[13] E.J. Eichten and C. Quigg, Phys. Rev. D49 (1994) 5845.

[14] V.V. Kiselev, Nucl. Phys. B406 (1993) 340;

P. Colangelo, G. Nardulli, and N.Z. Paver, Z. Phys. C57 (1993) 43;

T.M. Aliev and O. Yilmaz, Nuovo Cim. 105A (1992) 827;

C.A. Dominguez, K. Schilcher, and Y.L. Wu, Phys. Lett. B298 (1993) 190;

S. Reinshagen and R. Ruckl, Proceedings of 28th Rencontres de Moriond: QCD and High Energy Hadronic Interactions, Les Arcs, France, 20-27 Mar 1993. p. 443 ;

M. Chabab, Phys. Lett. B325 (1994) 205.

[15] L. Clavelli, Phys. Rev. D26 (1982) 1610;

Ch-R. Ji and F. Amiri, Phys. Rev. D35 (1987) 3318;

C.-H. Chang and Y.-Q. Chen, Phys. Rev. D46 (1992) 3845, Phys. Lett. B284 (1992) 127;

E. Braaten, K. Cheung, and T.C. Yuan, Phys. Rev. D48 (1993) 5049, hep-ph/9305206;

V.V. Kiselev, A.K. Likhoded, and M.V. Shevlyagin, Z. Phys. C63 (1994) 77;

Y.-Q. Chen, Phys. Rev. D48 (1993) 5181. 
[16] F. Abe et al. CDF Collaboration, FERMILAB-Pub-97/058-E, 1997.

[17] M.S. Alam et al. CLEO Collaboration, Phys. Rev. Lett. 74 (1995) 2885.

[18] W. Adam et al. DELPHI Collaboration, CERN-PPE/96-119, 1996.

[19] G. Alexander et al. OPAL Collaboration, Z. Phys. C70 (1996) 197.

[20] P. Abreu et al. DELPHI Collaboration, CERN-PPE/96-194, 1996.

[21] R. Barate et al. ALEPH Collaboration, CERN-PPE/97-026, 1997. 